\title{
La traducción de verso inserto en prosa: dos casos
}

\section{Bianchinetta Benavides Segura $^{1}$}

Universidad Nacional, Costa Rica

\section{RESUMEN}

Se describen los resultados de la aplicación del denominado "tratamiento completo", en la traducción de dos fragmentos poéticos insertos en prosa no literaria. Se examina la deconstrucción del texto original, sugerida como resultado de un análisis teórico que destaca en la importancia del componente cultural y la función apelativa inherente como la base de las soluciones traductológicas.

\section{Abstract}

A description is provided of the resulting application of the so-called complete treatment for the translation of two poetic fragments inserted in nonliterary prose. The suggested deconstruction of the source text, resulting from a theoretical analysis, is examined: thus the emphasis on the importance of both the cultural component and the inherent appellative function as a basis for different solutions for translation problems.

Palabras clave: Traducción, poesía. folclor, música. Keywords: Translation, poetry, folklore, music.

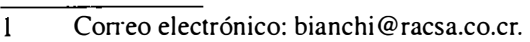


Si yo he logrado ver más allá que otros, es porque me he apoyado sobre hombros de gigantes.

Isaac Newton

En un estudio previo ${ }^{2}$ se examinaron los fundamentos teóricos utilizados en la metodología denominada el tratamiento completo creada para la traducción de los versos insertos dentro de la prosa del libro North Carolina and Old Salem Cookery ${ }^{3}$. A continuación se analizan dos casos de su aplicación.

El tratamiento completo intenta rescatar la intención de los fragmentos insertos en un macrotexto; este procedimiento, que consta de doce pasos, se puede subdividir en tres etapas. Una primera que incluye la identificación, la separación y la correspondiente contextualización de los fragmentos en el texto original (T.O.). La segunda que analiza la correspondencia del fragmento con la función de la traducción y su valor en el poema de origen; y una etapa final en la cual se establece la forma definitiva que tomará el fragmento equivalente en el texto terminal (T.T.) a fin de rescatar su relación rítmica, sintáctica y semántica con el resto de la traducción del poema de origen. Producto de esta recreación traductológica, se inserta un fragmento de un nuevo poema no solo en la misma posición que tenía el original sino con una función apelativa equivalente.

\section{Primer caso: Villancico Morning Star}

El primer paso consiste en la identificación del poema de origen $^{4}$ de donde se extrajo el fragmento. Bajo el subtítulo "Morning

$2 \quad$ Bianchinetta Benavides, "El tratamiento completo: una alternativa para la traducción del verso inserto en prosa no literaria." Letras 39 (2006) 183-203.

3 Beth Tartan, North Carolina and Old Salem Cookery (Chapel Hill: The University of North Carolina Press, 1992).

4 La tipografía cursiva hace referencia a los nombres dados a los distintos pasos del proceso. 
Star", en el Capítulo vi, aparecen las dos primeras líneas de la letra del villancico con el mismo nombre, de la siguiente manera:

Morning Star, Oh cheering sight, Ere thou camest, how dark Earth's night...

Este villancico, compuesto por Francis F. Hagen entre 1844 y 1854 y traducido del alemán al inglés por Martin Houser en 1907, corresponde a la tradición religiosa de la Iglesia Morava, propia de los antiguos colonos de Old Salem, quienes llegaron a Carolina del Norte procedentes de Alemania a mediados del siglo xvII trayendo consigo sus costumbres y prácticas religiosas. En la actualidad este villancico forma parte de la colección de cantos navideños de la práctica presbiteriana de varias congregaciones de distintos estados del sur; de hecho, su partitura se obtuvo en una visita a la Tercera Iglesia Presbiteriana del condado de Hunstville, Alabama. La interpretación de este villancico es factible escuchando Joining our Voices ${ }^{5}$ y el texto completo se puede obtener ingresando a distintos sitios en la internet ${ }^{6}$ pertenecientes a organizaciones que patrocinan el rescate de las tradiciones moravas. Veamos el texto original completo:

Morning Star, O cheering sight

Ere Thou camst how dark earth's night

Jesus, mine, in me shine

Fill my heart with light divine.

Morning Star, Thy glory bright

Far excels the sun's clear light:

Jesus, be constantly.

More than thousand suns to me

$5 \quad$ Moravian Music Foundation, Joining our Voices (P.O. Box, Salem Station, Winston-Salem, NC 27108); 2003.

6 Por ejemplo: <www.moravianmusic.org $>$. 
Thy glad beams, Thou Morning Star,

Cheer the nations near and far.

Thee we own, Lord alone

Man's great Saviour, God's dear Son

Morning Star, my soul's true Light,

Tarry not dispel my night

Jesus, mine, in me shine

Fill my heart with light divine.

Concluida la ubicación del fragmento, se procedió a establecer su relación con el resto del poema. Lo primero es que el fragmento inserto corresponde a los dos primeros versos del villancico los cuales además de rimar entre sí, inician el patrón de rima consonante presente en todo el canto. Además la frase Morning Star aparece en el primer verso de cada estrofa y que los últimos lexemas de los dos primeros versos de este fragmento tienen rima idéntica a la de los dos primeros versos de la segunda y cuarta estrofas, por lo que se procura rescatar esta relación en el T.T.

Al corroborar los significados de cada una de las palabras, se debe considerar que un verso religioso en sí mismo es una reelaboración de textos procedentes de la Biblia. Desde las perspectivas cristiana y judía, los versos de inspiración bíblica encierran una gran variedad de aspectos referentes los contextos culturales varios en que se originaron y se practican. Las referencias bíblicas dentro de la poesía pueden aparecer ya sea brevemente enunciadas o como parte del fragmento de un texto independiente; por tanto, su traducción considera las generalidades de forma y connotación particulares y relevantes a un subgénero independiente. La proximidad de los contextos religioso y cultural sugiere reevaluar varias consideraciones teóricas al respecto. Para lograr una versión comprometida con la intencionalidad del T.O., hay que recordar que la letra de los cantos religiosos son adaptaciones inspiradas en textos escritos siglos atrás en 96 
una lengua y cultura muy distintas. Las obras maestras de la literatura religiosa, como la Biblia y el Corán, son traducciones de traducciones realizadas ante la necesidad de llevar las doctrinas inscritas en estas obras a culturas de distintas lenguas. Al consultar muchos textos paralelos utilizados en el contexto religioso de la cultura terminal (El villancico Oh Aldehuela de Belén), su estructura apunta al rescate de un tono solemne incluso en los casos de lexemas que carecen de consonancia en la rima. Además, su traducción al castellano mantiene los recursos textuales que producen no solo un efecto de fervor y solemnidad sino que rescatan una forma poética con emotividad y naturalidad funcional.

El cuarto paso corresponde a la elaboración de una traducción lineal de todo el poema de origen en aras de su reelaboración final a partir de una estructura primaria semejante en orden y cantidad de palabras; veamos:

Estrella de la mañana, oh vista alegre qué oscura la noche en la tierra, si tu no llegas Jesús mío en mi brillas Llena mi corazón con luz divina Estrella de la mañana, brillante gloria Por mucho superas la luz clara del sol Jesús se constantemente Más de mil soles para mí Tus alegres rayos, estrella de la mañana, Alegra las naciones cercanas y lejanas.

Sólo a Ti te debemos Señor al gran Salvador del hombre, el amado hijo de Dios

Estrella de la mañana, luz verdadera de mi alma qué oscura la noche en la tierra, si tu no llegas Jesús mío en mi brillas Llena mi corazón con luz divina 
El siguiente paso define la carga semántica de cada lexema al clasificarlos según sean de primer, segundo o tercer orden ${ }^{7}$, siendo los primeros imprescindibles en la traducción. Los lexemas de segundo orden se conservan en aras al rescate del ritmo y la rima, mientras que los de tercer orden se movilizan con más facilidad, y resultan incluso prescindibles. De este proceso surge la siguiente clasificación:

Lexemas de primer orden:

Morning Star, dark night, mine, in, shine, light, divine (en la primera estrofa) thy, bright, sun, light, Jesús, more, suns (en la segunda estrofa)

thy, beams, Morning Star, cheer, nations, God's, son (en la tercera estrofa)

soul's, true, Light, my, night, mine, shine, light, divine (en la cuarta estrofa)

Lexemas de segundo orden:

thou, camst, Jesús, me, in, fill, heart (en la primera estrofa)

Morning Star, glory, excels, be, constantly, thousand (en la segunda estrofa)

far, Lord (en la tercera estrofa)

my, dispel, Jesus, me, fill, Heart (en la cuarta estrofa)

Lexemas de tercer orden:

cheering, sight, earth's, how (en la primera estrofa)

far, clear, than (en la segunda estrofa)

glad, thou, near, and, dear, great, man's, saviour, thee, own, alone (en la tercera estrofa)

Morning Star tarry, not, with (en la cuarta estrofa)

7 Benavides. 183-203. 
Se procede a identificar la relación sintáctica entre los versos, considerando los tres tipos de lexemas dentro de los sintagmas verbales, lo que conduce a la relación entre el primer y el segundo verso y el tercero y cuarto de todas las estrofas, así como la función adjetivo-calificativa de las frases nominales al final del los cuatro primeros versos. Posteriormente se retorna a los lexemas para encontrar sinónimos para los lexemas de primer orden cuya acentuación y número de sílabas contribuyen a mantener el ritmo del poema.

Lo siguiente es reubicar los lexemas con el propósito de mantener la rima y conservar de una forma rítmica, rimada y con formas verbales y pronominales solemnes, invocaciones prototípicas, y vocabulario específico en forma de versos con naturalidad y efecto apelativo. Luego se cuenta las sílabas de los versos traducidos para verificar la correspondencia con el número de golpes y acentos rítmicos de la partitura, conteo que se le ha realizado al T.O. antes de su traducción lineal.

Las decisiones traductológicas que resulten de los pasos octavo y noveno deben apuntar no sólo a mantener el número de sílabas de los versos y su correspondencia con los golpes de la música, sino a rescatar la función apelativa, la forma rítmica, el efecto de la rima y la connotación religiosa del villancico. Por esa razón, tenemos casos como el de la traducción del verso Thee we own, Lord alone, Man's great Saviour, God's dear Son, de 13 sílabas, cuya versión lineal sería Sólo a Ti te debemos Señor, el gran Salvador del hombre, el amado Hijo de Dios, con 26 sílabas e imposible correspondencia musical. Por eso, más allá de una simple búsqueda de sinónimos, estos cambios de orden y selección terminológica se realizaron en aras del rescate de la correspondencia musical. La frase Thee we own, Lord alone, pasó por al menos tres posibles traducciones. La primera es Te reconocemos $o$ Te confesamos, único Señor o sólo a Ti, Señor. Esta última es literalmente correcta aunque impide la métrica requerida. La segunda fue Tú nos perteneces, único Señor, la cual resultó carente de sentido en español desde la perspectiva del cristianismo tradicional. Otra 
posible traducción sería Nosotros Te pertenecemos acompañada de sólo a Ti, Señor (parecida a la estructura denotativa de la primera). En la versión final, sin embargo, nos inclinamos por la frase Todo el orbe te es siempre fiel $^{\$}$, en la cual se cambiaron casi todas las categorías sintácticas con el propósito de rescatar la consonancia de la rima y la correspondencia del verso con el ritmo musical. Otro caso aparece en el cuarto verso de la tercera estrofa, Man's great Saviour, God's dear Son, que se tradujo como Oh Hijo de Dios, Gloria de Israel. Prescindimos del término querido asociado al de Hijo por resultar carente de rima y correspondencia con la duración del tiempo de la música. Otras opciones como Cristo Redentor, que sí concordaban con la música, no mantenían la consonancia de la rima entre las palabras son y alone del inglés. Por eso se optó por traducirlo como Gloria de Israel $^{9}$ para que coincidiera con la rima de Todo el orbe te es siempre fiel.

A fin de procurar la correspondencia entre la versión en castellano y la música compuesta para el villancico original fue necesario atender tres aspectos. Primero, al final de cada línea se establece la necesidad de buscar una palabra aguda (i.e. matinal, celestial, amor, señor, etc.). En el texto original, una buena parte de las palabras finales son monosilábicas. Otras tienen una sílaba final formada por fonema consonante precedido por vocal (i.e. alone, divine). La estructura silábica del T.O. debe coincidir con la del T.T, de lo contrario se pierde la correspondencia musical. Segundo, la música está escrita en un compás de cuatro tiempos, que corresponde a cuatro notas negras o el doble de notas corcheas. En este compás, los tiempos primero y tercero son acentuados. Estos acentos se deben corresponder con los acentos de las palabras del texto, para que haya naturalidad en las frases al momento de cantarlas. Por ejemplo: Qué oscura es la noche $\sin \mathrm{Tu}$ brillo celestial. Tercero, en el texto original varias palabras se

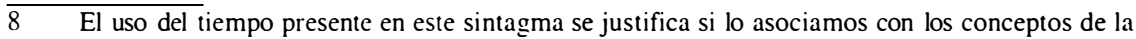
creación y la eternidad que según la Biblia, obedecen al tiempo de Dios. sin pasado. presente o futuro sintáctico. (Referencias: Proverbios 16,4; Isaías 47, 7-9: Mateo 6, 27; Mateo 10. 30-31: Efesios 3, 20; Lucas 12. 22-31). Tomado del Cántico de Simeón. Evangelio de Lucas 2, 29-32. 
dividen para que a cada una de sus sílabas fonéticas le correspondan dos notas, usualmente corcheas, por ejemplo: ere thou camst how dark earth's night. En el texto en español, se optó por una correspondencia de una sílaba por cada nota. Este recurso amplía el margen de los criterios para la selección de sinónimos y la construcción de las frases; esto fue de bastante ayuda en el proceso. El ejemplo correspondiente es qué oscura es la noche sin Tu brillo celestial, en el cual se mantienen las doce notas del original. Veamos:

E ere tho u ca amst ho ow da ark $e$ arth's night Queos cura esla no che sin tu bri llo ce les tial

En algunos casos, se modificó el ritmo o la melodía para ajustarla al nuevo texto. Por ejemplo, la frase Jesus be, constantly se podría traducir Eres Tú, Jesús, constantemente pero, como vemos a continuación, requiere de una nota más para ser cantada, lo cual implicaría dividir la última y convertir la correspondiente negra en una corchea con puntillo y una semicorchea:

$\begin{array}{lcccccccccc}\text { Je } & \text { e } & \text { su } & \text { us } & \text { be } & \text { co } & \text { ons } & \text { ta } & \text { an } & \text { tly } & \\ \mathrm{e} & \text { res } & \text { tú } & \text { Je } & \text { ús } & \text { co } & \text { ons } & \text { tan } & \text { te } & \text { men te }\end{array}$

Esta estrategia no se siguió en esta traducción, ya que para este verso en particular, al final se utilizó otra frase que mantuviera la consonancia de los versos. Sin embargo, la misma se sugiere como un recurso alternativo para la traducción de otros textos similares.

Los cambios finales de orden sintáctico y terminológico llevaron a colocar el verso More than thousand suns to me del cuarto al tercer verso de la segunda estrofa de la misma forma que en los casos de Thee we own, Lord alone y Man's great Saviour, God's dear Son. $\mathrm{Y}$ de esta forma lograr una rima asonante. Otro ejemplo de reordenamiento sintáctico lo vemos en la traducción de Morning Star, My soul's true. Aquí, en lugar de Oh buen Jesús, eres la luz de mi alma, 
se prefirió Oh buen Jesús, de mi alma la luz. Con esta sinéresis, se logra no sólo la coincidencia entre las vocales al final de cada verso, sino la posibilidad de unirlas al momento de ser cantadas.

Una vez concluida esta etapa, se debe verificarla correspondencia del ritmo del poema con el de la música. Para esto utilizamos programa informático Cakewalk Express $^{10}$, utilizado por músicos profesionales para componer y hacer arreglos musicales, que permite escuchar la música al mismo tiempo que despliega en la pantalla las notas de la partitura original a la cual le hemos agregado nuestra traducción al español, lo que lleva a afirmar que la siguiente versión tiene total correspondencia con la música del villancico:

Oh Jesús, estrella matinal

¡Qué oscura es la noche sin tu brillo celestial!

En mi corazón brilla mi Señor

¡Lléname con tu resplandor!

Luz sin par, tu brillo de amor

$\mathrm{Ni}$ el Sol en su grandeza te supera, Oh Señor

Eres tú Jesús, más que soles mil, ven y quédate junto a mí

Tu fulgor, estrella matinal, alegra las naciones y las aleja del mal Oh Hijo de Dios, Gloria de Israel ¡Todo el orbe te es siempre fiel!

¡Oh Jesús de mi alma eres la luz!

¡Qué clara es mi noche si no demoras Tú!

En mi corazón brilla mi Señor

¡Lléname con tu resplandor!

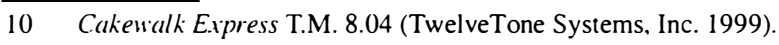


Concluido este proceso de traducción y edición, se procede a insertar el fragmento poético en el T.T. analizando cuidadosamente la forma del nuevo fragmento poético y las justificaciones que apoyen su reaparición. Pese a que la autora nos presenta una versión segmentada del poema, se ha realizado la traducción del poema completo que rescata el valor de figuras propias del contexto, con las que se siente emotivamente identificado. Nuestra versión ha puesto especial atención a las especificaciones que los teóricos sugieren para lograr traducir la musicalidad del texto poético ${ }^{11}$.

\section{Segundo caso: Poema Andy Gould's Mill}

En Capítulo III de North Carolina and Old Salem Cookery, Beth Tartan insertó el poema popular folclórico titulado "Andy Gould's Mill" en la sección de su libro titulada Water-Ground Cornmill, referente a la tradición de cosecha y tratamiento del maíz. La identificación del poema de origen, lo realiza la autora misma quien indica que escrito en 1897 por James Larkin Pearson, poeta oriundo de Wilkes, y quien durante veinte años, fue editor de la revista The Fol-Killer, cuyo material, escrito en dialecto rural del sur, trata del paisaje y la vida diaria del campesino. Este poema aparece con la siguiente forma:

The poem is about Andy Gould's mill, located in Wilkes County near Moravian Falls before it was inundated by the water of the Yadkin River many years ago ${ }^{12}$.

My Maw she said the meal was outce

Thar weren't another dust

She coudn't bake no supper bread

With brown an' crunchy crust

\footnotetext{
11 Esteban de la Torre. "Problemas de la traducción literaria". Teoría de la Traducción Literaria (Madrid: Síntesis, 1994) 209.

12 Tartan, 45.
} 
My Paw he shell a turn o' corn

An' poured it in a sack

An' off to Andy Gould's Mill

I toted jist a little turn.

Bekaze I was so small

An'brother Joln weren't big enough

To tote no turn at all

But he jist went along with us

As little fellows will,

To cross the river in the boat

At Andy Gould's Mill.

Se procedió a corroborar los significados de las palabras, poniendo especial atención en el contexto regional las culturas de origen y de llegada, encontrando así elementos comunes como el río, el molino, el bote y el maíz, así como las figuras del campo, el dialecto rural, de la mamá que cocina en casa y del papá quien debe traer el maíz.

La traducción lineal del poema supuso al menos doce versiones con distintas infracciones justificables en función de la búsqueda de una correspondencia analítica, ya que al tratarse de versos folclóricos, en algunos casos se apuntó a la connotación universal de las figuras; en otros, a la búsqueda del efecto de la rima; en otros, a la identidad o pertenencia a una cultura determinada; mientras que en otros, al apelativo sabor campesino. La clasificación de los lexemas según su carga semántica nos lleva a la siguiente organización:

Lexemas de primer orden: my, maw said, meal, couldn't, bread, brown, crunchy, paw, corn, poured, sack, Andy Gould, mill, I, turn, brother John, river, boat.

Lexemas de segundo orden: out, bake, an' o', toted, bekaze, big enough, no turn at al, to cross 
Lexemas de tercer orden: she, another, dust, supper, crust, turn, shell, An' off to, jist, little, fellows.

El proceso de rescate de lexemas nos orientó en distintas direcciones, dado que en ocasiones ha sido necesario aislar los elementos del T.O, deseamos rescatar su impacto ${ }^{13}$. Por ejemplo, la mención al tote en funciones intransitiva e infinitiva en los versos 8 y 11 del T.O. respectivamente, corresponde al saco utilizado en las zonas rurales de los Estados Unidos para cargar el maíz al hombro. Al inicio, se consideró la opción de utilizar el lexema alforja por ser el término cultural más equivalente. Sin embargo, como se dificultaba la conservación de la rima, se empleó saquillo y saco respectivamente en sustitución de ambas menciones. Otro caso fue la necesidad de modulación de elementos como supper en función calificativa de bread, debido a que en la cultura meta el pan se consume a toda hora y no solo en la cena. En el caso de los lexemas crunchy, bread, small, se tradujeron como tostadito, pancito y pequeñito con el fin de incorporar el uso del diminutivo, tan común en el dialecto regional meta. También se incorporaron los lexemas regionales mama y tata en sustitución de los lexemas Maw y Paw y se procuró el rescate del carácter melodístico del folclor, por su condición imprescindible y esencial $^{14}$, al sustituir formas regionales como thar y bekaze con una reordenación de lexemas de otros versos.

Por tratarse de una estrofa de quince versos, su relación sintáctica se establece más bien en función de una reubicación que rescate la rima. Este poema presenta cuatro núcleos temáticos comprendidos entre los versos uno y cuatro; cinco y ocho; nueve y once; y trece y quince. Dado que en castellano suele usarse más palabras para expresar la misma idea, la reconstrucción total del poema amplía su número

13 Christiane Nord, Text Analysis in Translation (Amsterdam: Editions Rodopi, 1991) 30.

14 Esteban de la Torre. "Problemas de la traducción literaria," Teoría de la Traducción Literaria (Madrid: Síntesis, 1994) 207. 
de versos a veintiuno, producto de la gran presencia de interjecciones y de elementos sintácticos y semánticos propios del registro regional del sur. Para mantener el tono coloquial de los primeros cuatro versos, se divide el primer verso My Maw she said the meal was out en dos sintagmas verbales, se rescata el tono regional de los lexemas del segundo verso, luego se invierte el orden de los elementos de los versos tres y cuatro, para incluir Que nuestra delicia sería, quedando así una sección equivalente de cinco versos. Este mismo procedimiento de división e inversión sintáctica se realiza con los siguientes tres versos, para agregar muy campante lo llevaba, con el fin de compensar varias elipsis. La extensión sintáctica más significativa se realiza al final del poema, cuando se agrega los versos $Y$ tan crecido que estaba y casi a rastras nos llevaba, que hacen alusión al río Yadkin antes de la inundación que acabó con el molino, según se explica en la introducción al poema.

Este proceso nos lleva a realizar más cambios finales de orden sintáctico y terminológico, con el fin de reflejar la motivación del fragmento en el contexto pero sacrificando en esta oportunidad una variedad de elementos del texto de origen ${ }^{15}$. Así, otros cambios en el orden buscan una correspondencia semántica y funcional, por medio de versos frescos con rima consonante al inicio y asonante en el resto del poema. Otro cambio fue incluir la interjección $O h$ en los versos primero y sexto del poema en castellano, ya que por ser dialectalmente transferible de una lengua a otra, pretende reforzar el tono coloquial y folclórico de los versos que inician con los lexemas mama y tata.

Llegado el momento de insertar el nuevo poema en el T.T., la nueva versión muestra una emotividad que, lejos de ser circunstancial e histórica, hace referencia a valores familiares universales porque, pese a sus modificaciones denotativas sustanciales, mantiene la estratificación social del dialecto rural del sur reflejada mediante elementos como la acentuación grave que el campesino costarricense da

15 Basil Hatim y Ian Mason. Teoría de la traducción: una aproximación al discurso (Barcelona: Editorial Ariel. 1995) 23. 
a los lexemas estándar agudos mamá y papá. La nueva versión emplea varios recursos para mantener la esencia folclórica costumbrista, y rescatar así el impacto de una escena vívida, con ayuda de cambio de orden de las palabras y el uso de sinónimos ${ }^{16}$.

Veamos la versión final:

Oh mi mama, que decía que sin harina no podía hacer ese pancito, tan dorado y tostadito que nuestra delicia sería.

Oh mi tata y su saquillo, Que de puro maíz llenaba y al molino de Andy Gould muy campante lo llevaba Por ratos yo lo cargaba ¡Y vaya si el saco pesaba! porque siendo tan chiquillo Apenas si lo aguantaba.

Oh mi hermanillo Juanito Que por ser tan pequeñito Conforme el viaje avanzaba Solo en el río pensaba.

$\mathrm{Y}$ tan crecido que estaba apenas si el bote cruzaba y al molino de Andy Gould casi a rastras nos llevaba.

Concluido este análisis, es posible afirmar que el tratamiento completo efectivamente guía hacia un procedimiento sistemático en la traducción de poemas insertos, un subgénero literario con tantas

$16 \quad$ Peter Newmark, A Textbook of Translation (Nueva York: Prentice Hall, 1988) 79. 
variables requiere una metodología que las incorpore y analice según su contexto y función particulares. Esta metodología sistemática, pero a su vez flexible, encierra las generalidades teóricas al mismo tiempo que incorpora la experiencia personal como integrante de la cultura receptora de esta propuesta. La traducción de estos poemas debe considerar los elementos extratextuales de un texto cultural, dada su contribución a la transmisión del sentido subyacente del texto original y, al igual que en el caso de la traducción de poemas completos, su traducción debe rescatar forma, tono, estilo y todos aquellos elementos que contribuyen a la creación del sentido.

Las herramientas traductológicas y metodológicas expuestas no son poco habituales y con características que muchos traductores ni siquiera considerarían importantes. Sin embargo, hemos fundamentado que, en el acto de traducir poesía, habrá cambios dadas las diferencias estructurales y antecedentes culturales de las dos lenguas y de las personas involucradas. El tratamiento completo recuerda que la traducción no ha de ser el doble del T.O., sino un producto estéticamente aceptable y acorde con las exigencias del género. $\mathrm{Si}$ hemos de traducir poesía, nuestro trabajo dependerá del tacto, el gusto y el papel de críticos y creadores, con el fin de que la versión traducida no parezca traducción. La españolización de las versiones terminales de los versos va en busca de su fidelidad completa, al mismo tiempo que rescata la forma y estilo poéticos; aquí la estética y fidelidad van de la mano. Sin embargo, donde hay conflicto, igana la estética!

La poesía tiene una forma y estilo propios; su supervivencia es posible sólo si es comprendida. Debido a que cada época aporta algo nuevo al contexto de un poema, el traductor debe dividir y segmentar los términos al traducir y disponer de todo elemento afectivo que le permita comprender y transponer el sentimiento. El tratamiento completo lleva a discernir entre lo propio y lo ajeno: primero nos presenta las costumbres extranjeras arraigadas al quehacer cotidiano del T.O. Luego nos conduce a través de los patrones de permisibilidad traductológica con el fin de regular la ambigüedad entre lo autóctono y lo 
extranjero. Por último, reproduce el entorno del poema por medio de una justificación intuitiva que apunta al rescate de la función de los poemas.

Beth Tartan intenta establecer un vínculo entre su libro y su lector al situarlo en sus raíces, con el fin de apelar a su subconsciente c intentar que muestre aprehensión por su cultura. Sus asociaciones se han percibido diferentes, en algunos casos con un sentido de transposición más amplio debido al límite geográfico. Se ha transpuesto entonces la diferencia sociolectal entre la suya y la del lector de la traducción mediante un intercambio semiótico que agrupe los diferentes procesos de socialización y la variedad de textos y géneros discursivos, al tiempo que deja de lado el carácter impersonal en pro de la búsqueda del porqué de las ideas. Al género de la poesía hay que buscarle el código genético: hay que estudiarlo en relación con lo que dice, cómo lo dice, y lo que esto implica. Por tanto, se puede afirmar que sí vale la pena traducir la forma del discurso poético porque ésta es parte de dicho código. La traducción del folclor debe buscar el dinamismo de las estructuras fijas: la métrica, la rima consonante y el número de versos, para hacer vibrar al lector de la traducción, como en este caso, lo transporten a deleitarse con sabor regional logrado.

La traducción de poesía popular, especialmente si está inserta en un macrotexto, debe dar a conocer los valores semánticos con la ayuda de una metodología disciplinada, que convierta al traductor de poesía en creador de materia y sentido. Por esta razón, el uso de otros recursos traductológicos más convencionales, por ejemplo una nota al pie de página que resuma la estructura connotativa del poema, podrían constituirse en un impedimento. El traductor habrá de estar alerta ante la presencia de poemas, ya que en otros géneros se ha de suponer que no fueron colocaron allí al azar.

En textos narrativos de tipo literario es habitual encontrar referencias culturales y religiosas de diversa procedencia. En North $\mathrm{Ca}$ rolina and Old Salem Cookery, los poemas transportan al lector original con rumbo a un molino, de regreso a casa para cantar un 
villancico en Nochebuena, y a muchos otros lugares en donde se evoca y revive la idiosincrasia de dichas experiencias, en las cuales, no se puede ni hace falta reproducir cada detalle sino más bien el clima total del poema ${ }^{17}$. Por estas razones, no hay que subestimar la calidad literaria de un texto cultural ni las distintas consideraciones referentes a las tradiciones y vivencias personales del autor.

El uso de los recursos informáticos, lejos de agregar un elemento de complicación a la traducción de este género, su incorporación más bien contribuye a darle a los lexemas de este fragmento un sentido de dirección, movimiento y dinamismo mágicos y emotivos. Transportar la versión española del villancico hacia los caracteres musicales de un programa de software equivale a hacer una alusión a quienes comparten una canción y revive una experiencia placentera, al escucharla una y otra vez. La valorización dada al estudio de la sincronía musical de este villancico ha producido resultados diacrónicos que nos han llevado a investigar más acerca de la historia de la lengua y la cultura que lo originó. De esta forma se amplía una visión evolutiva a lo largo del proceso de investigación. Muchos de los elementos utilizados tanto en la traducción de los fragmentos poéticos como en la traducción del resto de T.O. son producto de ese crecimiento de nuestra visión referente a los intertextos propios del género.

Por todo esto, el tratamiento completo codifica una parte del conocimiento ya existente con respecto a la traducción de poesía y se presta como alternativa para quien desee traducir las rimas, el ritmo y los lexemas del género y las variables culturales de dos pueblos. Este procedimiento resalta la importancia de analizar con profundidad a fin de entender y descubrir el mensaje del texto original. Esta sistematización de lo ya expuesto y aceptado fundamenta la aplicación de todas aquellas consideraciones personales que en su momento parecían ser puramente intuitivas y subjetivas y de utilidad en apariencia irrelevante. Por eso insta a la experimentación, a la emotividad y al

17 Luis Alonso Schökel y Eduardo Zurro, La traducción bíblica: lingüística y estilística (Madrid: Ed. Cristiandad, 1977) 160. 
uso de herramientas traductológicas poco convencionales como parte de los elementos que contribuirán a la creación del producto final. A la gran experiencia de Octavio Paz, Umberto Eco, Peter Newmark, Eugene Nida y tantos otros expertos en el campo de la traductología se ha adjuntado, con mucho respeto, la propia experiencia, las motivaciones y vivencias que interpretan el mundo que nos rodea y que nos han permitido convertirnos en creadores, en poetas, $i$ en traductores! En conclusión, la traducción de estos poemas constituye posibilidades traductológicas; la metodología dependerá, en forma exclusiva, de la experiencia individual y de las motivaciones del traductor. 


\section{Estrella Matinal (Morning Star)}

Francis Florentine Hagen 1836 - Traducido por Biacchinetta Benavides 2005
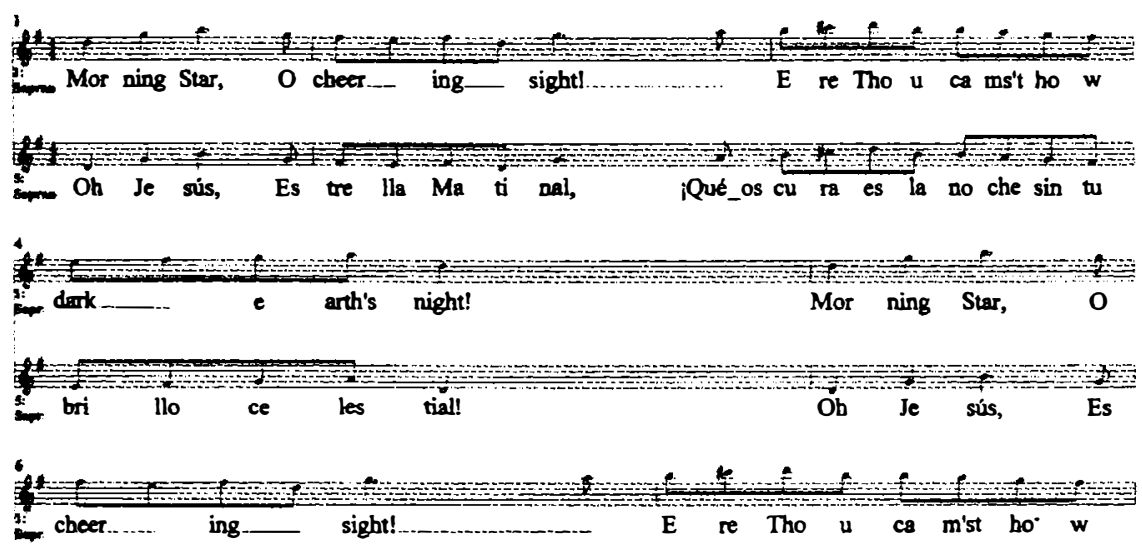

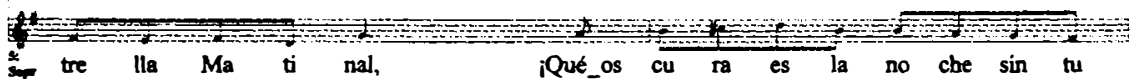

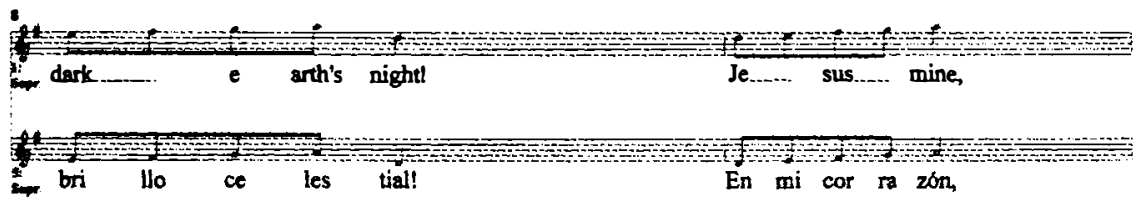

$\frac{\text { In }}{\text { In me... shine; me shine, }}$

240 ar. bri lla mi Se ñor; bri lla mi Se tor, en mi co ra zón;

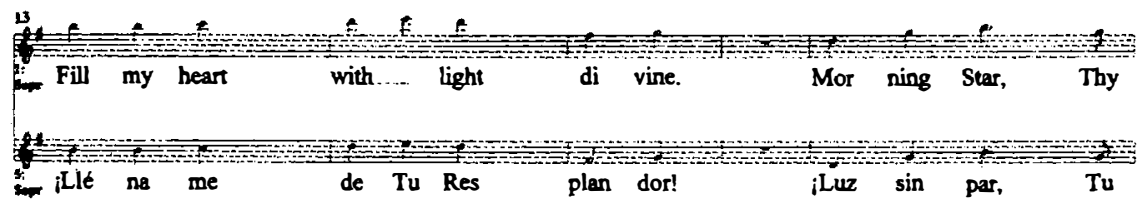


2 glo_ ry_.. bright.
bri llo de a mor! iNiel sol en su gran de za te su pe ra, oh Se bor!

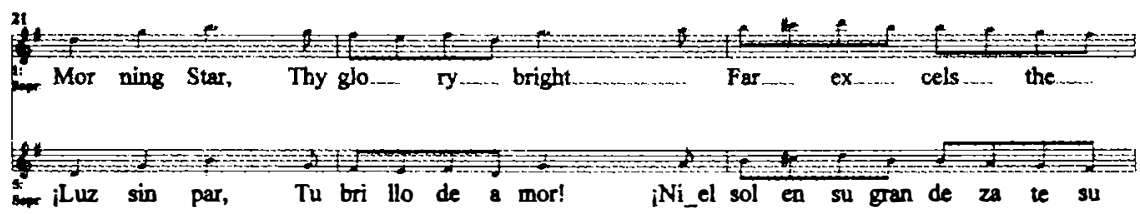
sun .. clear_- light;

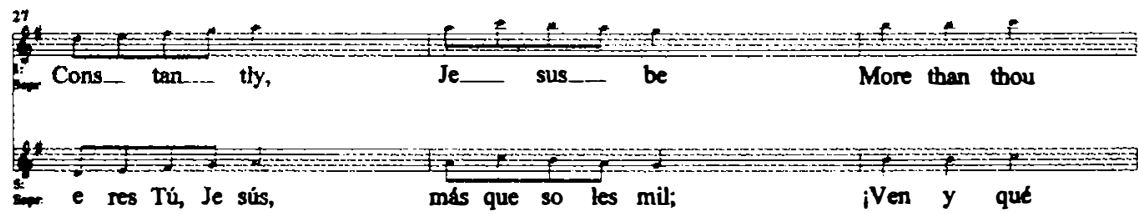
sand suns to me. Thy glad beams, Thou Mor.. ning Star,

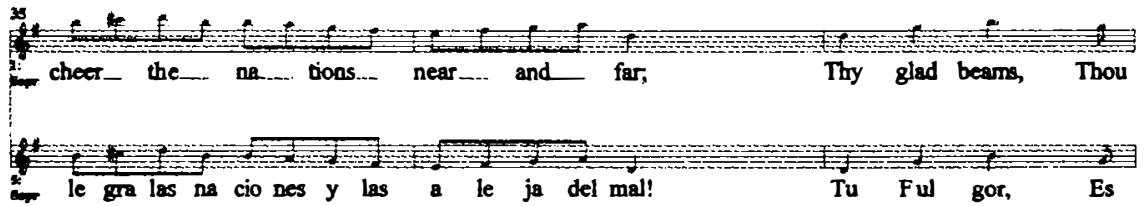



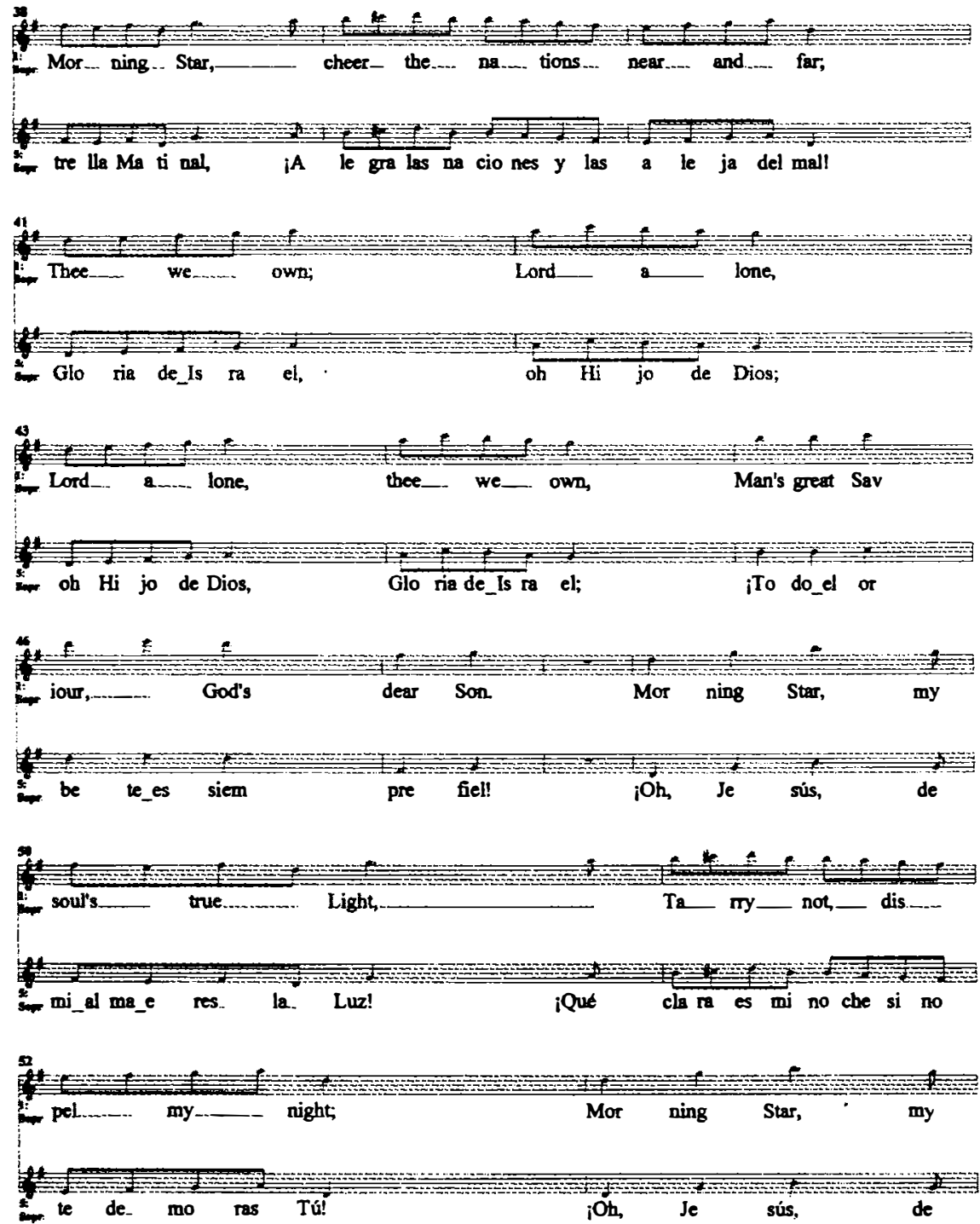
4

24. soul's__ true____.. Light,.................. Ta.... my._.. not,_... dis....

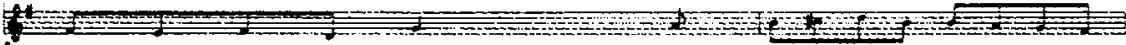
mi_al ma tes_ la Lual iQué cla ra es mi no che si no

6.

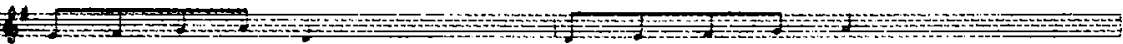
te de mo ras Tú! En mi co ra. zón

In 4100 bri lla $\mathrm{mi} \mathrm{Se}$ nor; bri lla mi Se for,

$\frac{d}{\text { Je_. sus...mine; }}$ Fill my heart L4. en mi co ra zón; jLlé na me de Tu Res plan dor! 